Mathematical Research Letters 1, 297-307 (1994)

\title{
INVARIANT ALGEBRAS AND COMPLETELY REDUCIBLE REPRESENTATIONS
}

\author{
H. KRAFT AND L. W. SMALL \\ To Peter Gabriel for his many contributions to algebra
}

\begin{abstract}
We give a general construction of affine noetherian algebras with the property that every finite dimensional representation is completely reducible. Starting from enveloping algebras of semi simple Lie algebras in characteristic zero we obtain explicit examples and describe some of their properties.
\end{abstract}

In the following an algebra $A$ will always mean an associative algebra over a field $k$ with a unit element. Mostly, we will assume that $A$ is affine and (left and right) noetherian. An $A$-module $M$ is a left $A$-module if not otherwise stated, and we will not distinguish between the module $M$ and the corresponding linear representation of $A$ on the vector space $M$.

\section{The condition FCR}

It is well known that the enveloping algebra $\mathfrak{U}(\mathfrak{g})$ of a semisimple Lie algebra $\mathfrak{g}$ in characteristic zero has the following two important properties:

- Every finite dimensional representation of $\mathfrak{U}(\mathfrak{g})$ is completely reducible.

- $\mathfrak{U}(\mathfrak{g})$ has enough finite dimensional representations, i.e., the intersection of the kernels of all finite dimensional representations is zero.

The first is due to H. Weyl, the second to Harish-Chandra (see [Dix] 2.5.7, p. 84). Algebras satisfying the second condition will be called residually finite-dimensional.

In the following we consider algebras $A$ which satisfy these two properties. For this purpose we introduce the following condition FCR (= "Finite dimensional representations are Completely Reducible"):

(FCR) $A$ is residually finite-dimensional and every finite dimensional representation of $A$ is completely reducible.

Received February 10, 1994. 
Beside the enveloping algebras and their factor algebras (and of course the finite dimensional semisimple algebras) the only other examples of FCRalgbras known to us are the quantum enveloping algebras $\mathfrak{U}_{q}(\mathfrak{g})$ for $q$ not a root of unity. Our first result shows that one can construct many more such algebras by taking invariants of FCR-algebras under finite groups.

Proposition 1. Let $B$ be a noetherian algebra satisfying condition FCR and let $H$ be a finite group of automorphisms of $B$ where $|H|$ and char $k$ are prime. Then the invariant algebra $B^{H}$ also satisfies FCR.

Proof. It is clear that $B^{H}$ is again residually finite-dimensional. Moreover, $B^{H}$ is a direct summand of $B$ as a left or right $B^{H}$-module. More precisely, every isotypic component $B_{\lambda}$ of $B(\lambda \in \hat{H}:=$ the set of isomorphism classes of irreducible representations of $H)$ is a two-sided $B^{H}$-module and $B=\oplus_{\lambda} B_{\lambda}$. This implies that for every $B^{H}$-module $W$ we have $W=$ $\left(B \otimes_{B^{H}} W\right)^{H}$ in a canonical way. Since $B$ is noetherian it follows from a result of Farkas and Snider (see [Mon] Chap. 5, Corollary 5.9) that $B$ and hence every $B_{\lambda}$ is finitely generated as (left or right) $B^{H}$-module.

Now let $W$ be finite dimensional. Then the kernel $I$ of the corresponding representation has finite codimension in $B^{H}$ and so the left ideal $B I \subset B$ also has finite codimension and is $H$-stable. As a consequence, the maximal two-sided ideal $J$ of $B$ contained in $B I$ satisfies the same properties. By assumption, $B / J$ is a semisimple algebra and since $I=(B I)^{H} \supset J^{H}$ we see that $B^{H} / I$ is a factor algebra of $(B / J)^{H}$ which is semisimple by a theorem of Levitzki (cf. [Mon] Chap. 1, Theorem 1.15; this also follows from our Lemma 2 below).

Remark 1. (a) It is already clear from the finite dimensional case that the result does not hold if we only assume that $A \subset B$ is subalgebra such that $B$ is finitely generated as an $A$-module. (Take the upper triangular matrices in the matrix ring.) However, it suffices to assume in addition that $A$ is a direct summand of $B$ as a (left or right) $A$-module.

(b) It would be of interest to find affine noetherian $k$-algebras $A$ in characteristic $p>0$ satisfying FCR. At present, we know of no such examples which are not finite dimensional.

(c) There are examples due to Montgomery and Small which show that the invariant algebra $A^{H}$ of an affine algebra $A$ need not be affine ([MoS] Example 2, page 38). But it is an open question whether affine and the property FCR implies that $A^{H}$ has again the property FCR. However, our proof above does not work in this case as shown by the following Example 1 the exposition of which is due D. S. Passman. We thank Passman for many useful conversations. 
Example 1. There exists a prime, residually finite-dimensional affine $k$ algebra A having an idempotent e such that some finite dimensional representation of $e A e$ is not the restriction of a finite dimensional representation of $A$.

Proof. Let $F=k\langle x, y\rangle$ be the free $k$-algebra in two variables and let

$$
A=\left(\begin{array}{cc}
k+F y & F \\
F y & F
\end{array}\right) \subseteq \mathrm{M}_{2}(F)
$$

where $F y$ is the left ideal of $F$ generated by $y$. Since $F$ is residually finite-dimensional, it follows that $A$ is also residually finite-dimensional. Furthermore, since $F$ is a domain, $A$ is easily seen to be prime, and we know that $A$ is finitely generated with generators $1, e_{12}, e_{22}, x e_{22}, y e_{22}$ and $y e_{21}$.

Notice that, for any integer $i \geq 0$, we have

$$
\left(\begin{array}{cc}
x^{i} y & 0 \\
0 & 0
\end{array}\right)=\left(\begin{array}{ll}
0 & 1 \\
0 & 0
\end{array}\right)\left(\begin{array}{cc}
0 & 0 \\
0 & x^{i}
\end{array}\right)\left(\begin{array}{ll}
0 & 0 \\
y & 0
\end{array}\right)
$$

Thus, if $\theta: A \rightarrow \mathrm{M}_{n}(k)$ is any homomorphism, then

$$
\theta\left(x^{i} y e_{11}\right)=\theta\left(e_{12}\right) \theta\left(\left(x e_{22}\right)^{i}\right) \theta\left(y e_{21}\right)
$$

In particular, since $\theta\left(x e_{22}\right)$ satisfies its characteristic polynomial, it follows that the sequence of matrices

$$
\theta\left(x^{0} y e_{1,1}\right), \theta\left(x^{1} y e_{1,1}\right), \theta\left(x^{2} y e_{1,1}\right), \ldots
$$

is linearly recursive.

Finally, if $e=e_{11}$, then $e A e \cong k+F y$ is easily seen to be the free algebra on the generators $x^{i} y$ for $i=0,1,2, \ldots$ Thus we can define a homomorphism $\theta^{\prime}: e A e \rightarrow k$ by $\theta^{\prime}\left(x^{i} y e_{11}\right)=1$ if $i$ is a power of 2 , and zero otherwise. In this case, it is obvious that the sequence

$$
\theta^{\prime}\left(x^{0} y e_{1,1}\right), \theta^{\prime}\left(x^{1} y e_{1,1}\right), \theta^{\prime}\left(x^{2} y e_{1,1}\right), \ldots
$$

is not linearly recursive and therefore $\theta^{\prime}$ cannot correspond to a composition factor of any such $\theta$ as above.

Let us recall another result in this context which which goes back to A. Weil (cf. Farkas [Far] and Lubotzky-Magid [LuM]). 
Proposition 2. If a finitely generated algebra A satisfies condition FCR then there are only finitely many non-equivalent representation in each dimension.

Outline of Proof (cf. $[\mathrm{LuM}])$. Consider the variety $\bmod _{A}^{n}$ of $n$-dimensional representations of $A$ (cf. [Kra2] Kap. II, $\S 2.7$ or [Kra1]). The group $\mathrm{GL}_{n}$ acts on $\bmod _{A}^{n}$ and the orbits are in one-to-one correspondence with the equivalence classes of $n$-dimensional representations. The condition FCR implies that all orbits are open. In fact, for every $M \in \bmod _{A}^{n}$ the normal space $T_{M}\left(\bmod _{A}^{n}\right) / T_{M}\left(O_{M}\right)$ is naturally embedded into $\operatorname{Ext}_{A}^{1}(M, M)$ where $T_{M}\left(\bmod _{A}^{n}\right)$ and $T_{M}\left(O_{M}\right)$ are the Zariski-tangent spaces at $M \operatorname{of}_{\bmod _{A}}^{n}$ and of the orbit $O_{M}$ of $M$.

Proposition 3. Let $k$ be a perfect field and $k^{\prime}$ any field extension of $k$.

(1) If $A, B$ are two FCR-algebras then $A \otimes_{k} B$ is FCR, too.

(2) The $k^{\prime}$-algebra $k^{\prime} \otimes_{k} A$ is FCR if and only if $A$ is.

Proof. Assertion (1) is obvious, and also one direction of assertion (2) is easy: If $k^{\prime} \otimes_{k} A$ is FCR then so is $A$.

For the other direction assume that $A$ is FCR. We first consider the case where $k^{\prime} / k$ is a finite extension. Then, by (1), $A^{\prime}:=k^{\prime} \otimes_{k} A$ is FCR as a $k$-algebra, hence also as a $k^{\prime}$-algebra.

Now let $k^{\prime}$ be the algebraic closure $\bar{k}$ of $k$ and put $\bar{A}:=\bar{k} \otimes_{k} A$. In this case the claim follows since every finite dimensional representation $\bar{V}$ of $\bar{A}$ is defined over a finite field extension $k^{\prime} / k$, i.e., there is a finite dimensional representation $V^{\prime}$ of $k^{\prime} \otimes_{k} A$ and an isomorphism $\bar{k} \otimes_{k^{\prime}} V^{\prime} \simeq \bar{V}$.

It remains to show that if both fields $k$ and $k^{\prime}$ are algebraically closed then every finite dimensional representation of $A^{\prime}:=k^{\prime} \otimes_{k} A$ is defined over $k$. This follows from the previous Proposition 2: The variety $\bmod _{A^{\prime}}^{n}$ is defined over $k$ and since it consists of finitely many orbits (= connected components) every such orbit is defined over $k$.

For a different proof see Remark 4 in Section 3.

\section{Invariants of enveloping algebras}

Interesting examples of FCR-algebras arise in the following way. Let $\mathfrak{g}$ be a semisimple Lie algebra (over an algebraically closed field $k$ of characteristic zero) and let $H$ a finite group of automorphisms of $\mathfrak{g}$. For example, we can take a finite subgroup of any semisimple group $G$ with Lie algebra $\mathfrak{g}$. Then $H$ acts on the enveloping algebra $\mathfrak{U}(\mathfrak{g})$ by means of algebra automorphisms. 
Proposition 4. The invariant algebra $A:=\mathfrak{U}(\mathfrak{g})^{H}$ has the following properties:

(1) A is affine, prime and (left and right) noetherian.

(2) A satisfies the FCR property.

(3) The center $\mathfrak{Z}(A)$ of $A$ is equal to $\mathfrak{Z}(\mathfrak{g})^{H}$ where $\mathfrak{Z}(\mathfrak{g})$ denotes the center of $\mathfrak{U}(\mathfrak{g})$. In particular, $\mathfrak{Z}(A)=\mathfrak{Z}(\mathfrak{g})$ in case $H$ is a subgroup of a semisimple group $G$ with Lie algebra $\mathfrak{g}$.

(4) A carries an ascending filtration such that the associated graded $\operatorname{gr} A=(\operatorname{gr} \mathfrak{U}(\mathfrak{g}))^{H}=\mathfrak{S}(\mathfrak{g})^{H}$ is commutative where $\mathfrak{S}(\mathfrak{g})$ denotes the symmetric algebra of $\mathfrak{g}$.

(5) $A$ is a maximal order and is Gorenstein see ([BeO] and [Bjo]).

(6) There is an action of $A$ on $A$ induced by the adjoint action of $\mathfrak{U}(\mathfrak{g})$ on $\mathfrak{U}(\mathfrak{g})$ such that $A$ becomes a direct sum of finite dimensional A-modules.

Proof. It is well known that $\mathfrak{U}(\mathfrak{g})$ carries an ascending filtration given by $\mathfrak{U}(\mathfrak{g})_{n}:=k+\mathfrak{g}+\mathfrak{g} \cdot \mathfrak{g}+\cdots+\mathfrak{g}^{n}$ whose associated graded algebra gr $\mathfrak{U}(\mathfrak{g})$ is the symmetric algebra $\mathfrak{S}(\mathfrak{g})$ of $\mathfrak{g}$. By assumption, the group $H$ respects the filtration, hence we get (4). The remaining claims follow immediately from this and Proposition 1 except assertion (3) which is a special case of the next lemma.

Lemma 1. Let $H$ be a finite group of automorphisms of $\mathfrak{U}(\mathfrak{g})$. Then $\mathfrak{Z}\left(\mathfrak{U}(\mathfrak{g})^{H}\right)=\mathfrak{Z}(\mathfrak{g})^{H}$.

(This lemma is also proved in [AlP, Proposition 1.4].)

Proof. This is a consequence of a theorem of Montgomery (see [Mon], Corollary 6.17) stating that $\mathfrak{Z}\left(B^{H}\right)=\mathfrak{Z}(B)^{H}$ in case $H$ consists of $X$ outer automorphisms. Recall that an automorphism is $X$-outer if it does not induce an inner automorphism on the total ring of quotients. So it remains to show that every $X$-inner automorphism $\sigma$ of $\mathfrak{U}(\mathfrak{g})$ is trivial. By definition we have $r \sigma(x)=x r$ for all $x \in \mathfrak{U}(\mathfrak{g})$ and a certain element $r$ of the total ring of quotients of $\mathfrak{U}(\mathfrak{g})$. Using the fact that the associated graded algebra gr $\mathfrak{U}(\mathfrak{g})$ has no zero divisors and is commutative we find that $\sigma$ respects the filtration and induced the trivial automorphism on $\operatorname{gr} \mathfrak{U}(\mathfrak{g})$. Thus $\sigma(\mathfrak{g})$ is a Lie subalgebra of $k \oplus \mathfrak{g}$, hence $\sigma(\mathfrak{g})=\mathfrak{g}$ and so $\left.\sigma\right|_{\mathfrak{g}}$ is the identity.

Example 2. Let $B=\mathfrak{U}\left(\mathfrak{s l}_{2}\right)$ and let $H=\mathbb{Z} / 2$ where the non-trivial element acts by conjugation with $\left(\begin{array}{cc}1 & 0 \\ 0 & -1\end{array}\right)$. Then $A:=B^{H}$ has GK-dimension 3 and has the same center as $\mathfrak{U}\left(\mathfrak{s l}_{2}\right)$, namely $\mathfrak{Z}(A)=\mathfrak{Z}(\mathfrak{g})=k[c]$ where 
$c$ is the Casimir element $c=h(h-2)+4 e f$, using the usual notation $h:=\left(\begin{array}{cc}1 & 0 \\ 0 & -1\end{array}\right), e:=\left(\begin{array}{ll}0 & 1 \\ 0 & 0\end{array}\right), f:=\left(\begin{array}{ll}0 & 0 \\ 1 & 0\end{array}\right)$.

We denote by $\bar{h}, \bar{e}$ and $\bar{f}$ the corresponding elements in $\operatorname{gr} B=\mathfrak{S}\left(\mathfrak{s} l_{2}\right)=$ $k[\bar{h}, \bar{e}, \bar{f}]$. Then $(\operatorname{gr} B)^{H}$ is generated by $\bar{h}, \bar{e}^{2}, \bar{f}^{2}$ and $\bar{e} \bar{f}$ and so $A$ is generated by $h, e^{2}, f^{2}$ and $e f$. It can be shown from this that the algebra $A$ has four non-equivalent irreducible representations in each dimension (see below for a general assertion). In particular, it is not a homomorphic image of an enveloping algebra of a semisimple Lie algebra.

Remark 2. (a) The picture is quite different in case the group $H$ is not finite. Let $H \simeq k^{*}$ be the maximal torus of $\mathrm{SL}_{2}$ acting by conjugation on $\mathfrak{s l}_{2}$. Then $A=\mathfrak{U}\left(\mathfrak{s l}_{2}\right)^{H}$ is commutative of dimension 2 , generated by $h$ and ef. (Again, gr $A=\mathfrak{S}\left(\mathfrak{s l}_{2}\right)^{H}=k[\bar{h}, \bar{e} \bar{f}]$ and $[h, e f]=0$ in $\mathfrak{U}\left(\mathfrak{s l}_{2}\right)$.)

(b) In a recent preprint Alev and Polo show that for any non-trivial finite group $H$ of automorphisms of $\mathfrak{U}(\mathfrak{g})$ the invariant algebra $\mathfrak{U}(\mathfrak{g})^{H}$ is never isomorphic to an enveloping algebra ([AlP $]$ Theorem 1) and also not to a factor algebra of an enveloping algebra if one assumes in addition that $H$ acts trivially on the center of $\mathfrak{U}(\mathfrak{g})$.

In order to calculate more examples we need the following results. Let $B$ be a $k$-algebra, $H$ a finite group of automorphisms of $B$ whose order is prime to the characteristic of $k$, and $A=B^{H}$ the invariant algebra. If $V$ is a finite dimensional simple $A$-module we denote by $I=I_{V}=\operatorname{Ann}_{A}(V)$ its annihilator ideal and by $H_{V}:=\left\{h \in H \mid h I_{V}=I_{V}\right\}$ the normalizer of $I_{V}$ in $H$. For simplicity we assume that $k$ is algebraically closed.

Proposition 5. There is a finite central extension $\widetilde{H_{V}}$ of $H_{V}$ which acts linearly on $V$, compatible with the action of $B$, such that $V$ has a decomposition of the form

$$
V=\oplus_{i=1}^{s} V_{i} \otimes W_{i}
$$

where the $V_{i}$ (resp. the $W_{i}$ ) are pairwise non-isomorphic simple $B^{H_{-}}$ (resp. $\widetilde{H_{V^{-}}}$) modules. In particular, the number s of blocks satisfies the inequality

$$
s \leq \text { \# irreducible representations of } H_{V} .
$$

(The compatibility of the two actions means that $h(b v)=\bar{h}(b) v$ for $h \in \widetilde{H_{V}}$, $\bar{h}$ the image of $h$ in $H_{V} \subset \operatorname{Aut}(B), b \in B$, and $v \in V$.)

For the proof we need the following easy lemma.

Lemma 2. Let $U=\oplus_{i=1}^{m} U_{j}$ be a finite dimensional vector space, $H \subset$ $\mathrm{GL}(U)$ a finite subgroup respecting the direct sum decomposition (i.e. $h U_{i}=$ 
$U_{\sigma(i)}$ with a permutation $\left.\sigma\right)$ and $H_{i}:=\left\{h \in H \mid h U_{i}=U_{i}\right\}, i=1, \ldots, m$. Then the projection $p_{i}: U \rightarrow U_{i}$ induces a surjective map $U^{H} \rightarrow U_{i}^{H_{i}}$.

Proof of Proposition 5. Consider $J:=\bigcap_{h \in H} h I$. Then $H$ acts by automorphisms on the semisimple algebra $B / J$ which contains as a factor $B / I_{V}=\operatorname{End}(V)$. Clearly, $H_{V}$ is the normalizer of this factor and so the lemma above implies that the canonical map

$$
p_{V}: B^{H} \rightarrow(B / J)^{H} \rightarrow\left(B / I_{V}\right)^{H_{V}}=\operatorname{End}(V)^{H_{V}}
$$

is surjective. It follows from Wedderburns theorem that the action of $H_{V}$ on $\operatorname{End}(V)$ is given by a homomorphism $H_{V} \rightarrow \mathrm{PGL}(V)$ which can be lifted to a homomorphism $\widetilde{H_{V}} \rightarrow \mathrm{GL}(V)$ where $\widetilde{H_{V}}$ is a central extension of $H_{V}$. The surjectivity of $p_{V}$ means exactly that the image of $B^{H}$ in $\operatorname{End}(V)$ is the centralizer of the image of $\widetilde{H_{V}}$. From this we immediately get the claimed decomposition of $V$. Since the kernel of $\widetilde{H_{V}} \rightarrow H_{V}$ acts by scalars on $V$ the number of non-isomorphic irreducible representations of $\widetilde{H_{V}}$ occurring in $V$ is at most the number of irreducible representations of $H_{V}$.

In order to apply the proposition above we say that a $B$-module $V$ admits a compatible action of $H$ if the annihilator $I:=\operatorname{Ann}_{B} V$ is stable under $H$ and the action of $H$ on $B / I=\operatorname{End}(V)$ is given by a linear representation of $H$ on $V$. Now the following corollaries are clear.

Corollary 1. Let $V$ be a finite dimensional simple $B$-module which admits a compatible action of $H$. Then the dimensions of the simple $B^{H}$ submodules of $V$ are the multiplicities of the irreducible subrepresentations of $H$ in $V$.

Corollary 2. (char $k=0$ ) Let $G$ be a simply connected semisimple group with Lie algebra $\mathfrak{g}$, let $H$ be a finite subgroup of $G$ and put $A:=\mathfrak{U}(\mathfrak{g})^{H}$. For every finite dimensional simple $\mathfrak{U}(\mathfrak{g})$-module $V$ we have a decomposition

$$
V=\oplus_{i=1}^{s} U_{i} \otimes M_{i}
$$

as an $A \times H$-module with pairwise non-isomorphic simple modules $U_{i}$ and $M_{i}$. Moreover, every finite dimensional simple $A$-module $M$ occurs in exactly one finite-dimensional simple $\mathfrak{U}(\mathfrak{g})$-module $V$.

(The last assertion follows from Lemma 1 because non-isomorphic $\mathfrak{U}(\mathfrak{g})$ modules have different central characters.)

With this corollary it is easy to verify the following examples. 
Example 3. Let $B=\mathfrak{U}\left(\mathfrak{s l}_{2}\right)$ and let $H=\mathbb{Z} / n$ where a generator of $H$ acts by conjugation with $\left(\begin{array}{ll}\zeta & 0 \\ 0 & 1\end{array}\right), \zeta:=e^{\frac{2 \pi i}{n}}$. Then $A_{n}:=B^{H}$ has GK-dimension 3 and has the same center as $\mathfrak{U}\left(\mathfrak{s l}_{2}\right)$, namely $\mathfrak{Z}(A)=\mathfrak{Z}(\mathfrak{g})=k[c]$. With the same notation as in Example 2 we find that $\operatorname{gr} A_{n}=(\operatorname{gr} B)^{H}$ is generated by $\bar{h}, \bar{e}^{n}, \bar{f}^{n}$ and $\bar{e} \bar{f}$. Thus $A$ is generated by $h, e^{n}, f^{n}$ and $e f$. It follows that the algebra $A_{n}$ has $n^{2}$ non-equivalent irreducible representations in each dimension.

Remark 3. In combination with Proposition 2 we obtain the following result: Let $H$ be a finite subgroup of a semisimple group $G(\operatorname{char} k=0)$. For any number $m$ there are only finitely many irreducible representations $V$ of $G$ which contain a simple $H$-module of multiplicity $\leq m$. In fact, we can show more:

There is a bound $N$ for the number of irreducible representations of $G$ containing a simple $H$-module with a given multiplicity.

As a consequence, we see that all examples of FCR-algebras constructed as above (by using finite subgroups of semisimple groups) satisfy the following property: There is a bound $N$ for the number of simple modules in any dimension. In particular, the function

$$
\tau_{A}(n):=\# \text { simple } A \text {-modules of dimension } \leq n
$$

has at most linear growth.

\section{The condition FPI and the Loewy-rank}

There are many another ways to express the condition FCR for an algebra $A$. One of them is related to polynomial identities. Denote by $I_{n} \subset A$ the ideal of polynomial identities of degree $n$, i.e. the ideal generated by the polynomial identities of $n \times n$-matrices, and consider the following condition:

(FPI) All ideals $I_{n}$ are of finite codimension.

(FPI stands for "Finite dimensional PI-factors.")

Proposition 6. An affine algebra A satisfies condition FPI if and only if there are only finitely many simple modules in each dimension.

Proof. Since every representation of dimension $n$ contains $I_{n}$ in its kernel it is clear that the first condition implies the second. For the inverse implication we use the fact that an infinite dimensional affine PI-algebra has infinitely many non-equivalent representations of a certain dimension $n$. This follows immediately from the Nullstellensatz which tells us that there 
are infinitely many maximal ideals which all have finite codimension (cf. [Row] Theorem 6.3.3).

With this result we get the following characterization of FCR-algebras.

Corollary 3. An algebra A satisfies condition FCR if and only if all factor algebras $A / I_{n}$ are finite dimensional and semisimple and $\bigcap_{n} I_{n}=(0)$.

Proof. One direction follows immediately from the above proposition and Proposition 2. For the other direction we first remark that each $I_{n}$ is the intersection of the maximal ideals containing it, because $A / I_{n}$ is finite dimensional and semisimple. Hence $A$ is residually finite-dimensional. Moreover, an $n$-dimensional representation of $A$ contains $I_{n}$ in its kernel and is therefore completely reducible.

Remark 4. From this result we get a different proof of Proposition 3(2): Let $A$ be FCR and let $V$ be a finite dimensional $k^{\prime} \otimes_{k} A$-module. Then $I_{n}$ is in the kernel of the map $A^{\prime}:=k^{\prime} \otimes_{k} A \rightarrow \operatorname{End}(V)$, hence the image $\bar{A}$ of $A$ in $\operatorname{End}(V)$ is finite dimensional and semisimple. It follows that the image of $A^{\prime}$ is a factor algebra of $k^{\prime} \otimes_{k} \bar{A}$ and therefore finite dimensional and semisimple, too.

The next result shows that the condition FPI carries over to invariant algebras under finite groups.

Proposition 7. Let $H$ be a finite group $H$ of automorphisms of an affine noetherian algebra $B$ where we assume that the order of $H$ is prime to the characteristic of the base field. Then the invariant algebra $B^{H}$ satisfies condition FPI if and only if $B$ does.

Proof. Clearly, for all $n \in \mathbb{N}$ the ideal $I_{n}$ of $A:=B^{H}$ is contained in the corresponding ideal $J_{n}$ of $B$. Therefore, $B / J_{n}$ is a finitely generated $A / I_{n^{-}}$ module and so $B / J_{n}$ is finite dimensional in case $A / I_{n}$ is. This proves one implication.

For the other implication assume that $B$ is generated, as an $A$-module, by $d$ elements. Let $M$ be a simple $A$-module and put $P:=B \otimes_{A} M$. Then $\operatorname{dim} P \leq d \cdot \operatorname{dim} M$ and $M$ occurs as an $A$-submodule of a JordanHölder-factor of $P$ as a $B$-module. This shows that every simple $A$-module is a Jordan-Hölder-factor of a simple $B$-module $\tilde{M}$ of $\operatorname{dimension} \operatorname{dim} \tilde{M} \leq$ $d \cdot \operatorname{dim} M$, viewed as an $A$-module. Thus, by Proposition $6, A$ satisfies FPI if $B$ does.

Another way to express condition FCR is based on the Loewy-rank of a finite dimensional module: It is equal to 1 if and only if the module is semisimple. (Recall that the Loewy-rank is the length of the series obtained 
by taking successively socles.) This condition was studied by Bell and Goodearl in $[\mathrm{BeG}]$.

Proposition 8. Let $A=B^{H} \subset B$ be as above. If every finite dimensional $B$-module has Loewy-rank $\leq m$ then the same holds for every finite dimensional A-module.

Let $M$ be a $B$-module with a compatible $H$-structure (see Section 2). Such a module admits an isotypic decomposition $M=\oplus_{\lambda \in \hat{G}} M_{\lambda}$ where each isotypic component $M_{\lambda}$ is a (left) $A$-module. Typical examples for modules with $H$-structure are the induced modules $B \otimes_{A} N$ where $N$ is an $A$-module and the $H$-structure is given by the $H$-action on $B$. Moreover, we obtain the isotypic decomposition of $B \otimes_{A} N$ in the following way:

$$
B \otimes_{A} N=\left(\oplus_{\lambda} B_{\lambda}\right) \otimes_{A} N=\oplus_{\lambda}\left(B_{\lambda} \otimes N\right) .
$$

Lemma 3. Let $N$ be an $A$-module and assume that $B \otimes_{A} N$ is a semisimple $B$-module. Then $N$ is semisimple, too.

Proof. Let $N^{\prime} \subset N$ be an $A$-submodule. Clearly, the submodule $\tilde{N}:=$ $B N^{\prime} \subset \tilde{M}:=B \otimes_{A} N$ is $H$-stable. We claim that it has a complement $P$ which is an $H$-stable $B$-module. It then follows that $P \cap N=P^{H}$ is a complement of $N^{\prime}$ in $N$ as an $A$-module.

In order to prove the claim we use the canonical linear map

$$
\operatorname{Hom}_{B}(\tilde{M}, \tilde{N}) \rightarrow \operatorname{End}_{B}(\tilde{N})
$$

which is surjective because $\tilde{M}$ is semisimple. It is easy to see that the map is $H$-equivariant where we use the usual action of $H$ on Hom and End. It follows that $\operatorname{Hom}_{B}(\tilde{M}, \tilde{N})^{H} \rightarrow \operatorname{End}_{B}(\tilde{N})^{H}$ is surjective, too.

Proof of Proposition 8. Let $N$ be an finite dimensional $A$-module and denote by soc $\tilde{N}$ the socle of the $B$-module $\tilde{N}:=B \otimes_{A} N$. This is a semisimple $H$-stable submodule. It follows from the lemma above that $(\operatorname{soc} \tilde{N})^{H}$ is a semisimple submodule of $N=\tilde{N}^{H}$. Hence, $(\operatorname{soc} \tilde{N})^{H} \subset \operatorname{soc} N$ and, by induction, we see that the Loewy-rank of $N$ is less or equal to the Loewy-rank of $\tilde{N}$.

\section{References}

[AlP] J. Alev, P. Polo, A rigidity theorem for finite group actions on enveloping algebras of semisimple Lie algebras, preprint (1993).

[BeG] A. D. Bell and K. R. Goodearl, Algebras of bounded finite dimensional representation type, preprint (1992). 
[BeO] M. van den Bergh and van Oystaeyen, Lifting maximal orders, Comm. Algebra 17 (1989), 341-349.

[Bjo] J.-E. Bjork, Filtered noetherian rings, Noetherian rings and their applications, Math. Surveys and Monographs, vol. 24, 1987.

[Dix] J. Dixmier, Enveloping algebras, North-Holland Publishing Company, Amsterdam-New York-Oxford, 1977.

[Kra1] H. Kraft, Geometric methods in representation theory, Representations of algebras, Workshop Proceedings (Puebla, Mexico 1980), Lecture Notes in Math., vol. 944, Springer-Verlag, Berlin-Heidelberg-New York, 1982.

[Kra2] _ Geometrische Methoden in der Invariantentheorie, Aspects of Mathematics, vol. D1, Vieweg Verlag, Braunschweig-Wiesbaden, 1985.

[LuM] A. Lubotsky and A. Magid, Varieties of representations of finitely generated groups, Memoires AMS 336 (1985), Providence, Rhode Island.

[Mon] S. Montgomery, Fixed rings of finite automorphism groups of associative rings, Lecture Notes in Math., vol. 818, Springer-Verlag, Berlin-Heidelberg-New York, 1980.

[MoS] S. Montgomery and L. W. Small, Fixed rings of noetherian rings, Bull. London Math. Soc. 13 (1981), 33-38.

[Pas] D. Passman, Infinite Crossed Products, Pure and Applied Mathematics, vol. 135, Academic Press Inc., Boston-San Diego-New York, 1989.

[Row] L. H. Rowen, Ring Theory, Student Edition, Academic Press Inc., Boston-San Diego-New York, 1991.

Mathematisches Institut Der Universität Basel Rheinsprung 21, CH-4051 BASEL, SWITZERLAND

E-mail address: kraft@urz.unibas.ch

Department of Mathematics, University of California, San Diego

LA Jolla, CA 92093

E-mail address: lwsmall@ucsd.edu 